\title{
Análise do movimento e desenvolvimento de um protótipo de cortador basal com discos bi-articulados ${ }^{1}$
}

\author{
Carlos A. A. Oliveira ${ }^{2}$, Oscar A. Braunbeck ${ }^{3} \&$ Carlos E. S. Volpato ${ }^{4}$
}

\begin{abstract}
RESUMO
O acompanhamento da superfície e a autoproteção, são fatores limitantes no mecanismo cortador de base convencional da canade-açúcar, acarretando corte e varredura ineficientes, além de promover a incorporação de elevada quantidade de terra ao material colhido. Um modelo de simulação foi desenvolvido por meio das leis da dinâmica para determinar os deslocamentos angulares do segmento no plano vertical da faca no plano horizontal e da força de interação do segmento com a superfície. A simulação do mecanismo foi realizada em duas velocidades (400 e 600 rotações min $^{-1}$ ), três alturas com relação à superfície $(120,130$ e $140 \mathrm{~mm})$ e duas inclinações do rotor $(-10$ e -12º). O resultado da amplitude de oscilação do segmento, variável relacionada à varredura, apresentou valores entre 2,1 e 9,50; a oscilação da faca apresentou variações angulares desprezíveis durante a simulação e a força de reação com a superfície, que está relacionada à movimentação de terra e danos à soqueira, apresentou valores entre 941 e 2654 N. Em função dos resultados, observa-se que recursos virtuais de simulação se apresentam como poderosa ferramenta na tomada de decisões e no desenvolvimento de novos mecanismos.
\end{abstract}

Palavras-chave: modelagem, simulação, mecanismos de máquinas agrícolas

\section{Analysis of movement and development of a prototype of a base cutter with bi-articulated discs}

\begin{abstract}
The accompaniment of the surface and the auto-protection are limiting factors in the sugar cane conventional base cutter mechanism which causes an inefficient cutting and sweeping and also promotes a high amount of soil incorporation to the harvested material. A simulation model was developed according to the Laws of Dynamics to determine the angular displacements of the segment in the vertical plan, the knife in horizontal plan and the force of interaction of the segment with the surface. The simulation of the mechanism was carried out in two speeds (400 and 600 rotations $\mathrm{min}^{-1}$ ), three heights with regard to surface $(120,130$ and $140 \mathrm{~mm})$ and two inclinations of the rotor $\left(-10\right.$ and $\left.-12^{\circ}\right)$. The reaction force on the contact surface, which is related to the sweeping, presented values between 21 and $95^{\circ}$. The oscillation of the knife presented insignificant angular variations during the simulation. The force of reaction with the surface, which is related to the soil movement and damages to the ratoons, presented values between 941 and $2654 \mathrm{~N}$. It was observed that virtual resources of simulation are presented as a powerful tool in decision-making and development of new mechanisms.
\end{abstract}

Key words: modeling, simulation, mechanisms of agricultural machines

1 Parte da Tese de Doutorado do primeiro autor, apresentada à FEAGRI /UNICAMP

${ }^{2}$ Faculdade de Agronomia, Universidade de Rio Verde - FESURV. C.P 104, CEP 75900-000, Rio Verde, GO. Fone: (64) 3620 2213. E-mail: carlosaao@fesurv.br

3 FEAGRI/UNICAMP. CEP 13083-970, Campinas, SP. Fone: (19) 3521-1049. E-mail: oscar@agr.unicamp.br

${ }^{4}$ DEG/UFLA. CEP 37200-000, Lavras, MG. Fone: (35) 3829 1669. E-mail: volpato@ufla.br 


\section{INTRODUÇÃO}

Uma das principais limitações das colhedoras de cana picada, maioria no Brasil, está relacionada ao mecanismo de corte, que se compõe de oito discos os quais apresentam, em suas extremidades, cinco facas fixas em cada rotor (Magalhães \& Braunbeck, 1998). Os discos se posicionam na entrelinha da cultura durante a operação de corte, não se adaptando ao sistema de plantio em sulcos utilizados no Brasil.

As perdas e a contaminação da cana-de-açúcar com terra durante o processo de colheita, estão associadas diretamente ao acompanhamento inadequado do perfil solo e da varredura ineficiente, realizadas pelo cortador basal (Volpato et al., 2002; Oliveira, 2003). Em certos casos, essas perdas chegam a $10 \%$ e as quantidades de terra incorporadas aos colmos estão entre 3 a $5 \mathrm{~kg} \mathrm{t}^{-1}$ (Braunbeck, 1999). A cana contaminada com impurezas durante a colheita reduz, ao ser processada na usina, as eficiências de filtragem e clarificação do caldo, parâmetros esses ligados à qualidade final do açúcar (Ridge \& Dick, 1992).

A redução de perdas e a contaminação com terra pelo cortador, requerem alterações neste mecanismo, haja vista que mudanças operacionais dos equipamentos existentes, sistematização dos terrenos não reduziram, de forma efetiva, a contaminação dos colmos ao longo da prática da colheita mecanizada (Braunbeck et al., 1999). A solução para este impasse tecnológico está na utilização de mecanismos passivos ou ativos, capazes de realizar um seguimento do perfil do solo e uma varredura das plantas acamadas de forma eficiente (Braunbeck \& Magalhães, 2002).

Ante este contexto, o desenvolvimento de protótipos virtuais tem sido uma ferramenta de grande sucesso e confiabilidade nos últimos anos, em função de oferecer a possibilidade de se verificar a viabilidade de um protótipo por meio de simulações, sob certas condições de operações, sem ter que construí-lo, evitando custos com projetos inadequados (Oliveira et al., 2005). O procedimento de simulação faz uso de técnicas de projeto de máquinas assistido por computador (Cad), de técnicas de programação convencional com uso de programas de computação simbólica e numérica (Matlab, Mathematica, entre outros) e programas computacionais específicos (Ansys, Adams, Working Model, entre outros) as quais são utilizadas de forma individualizada ou conjuntamente.

Objetivou-se, com este trabalho simular o desempenho do movimento de um protótipo de corte de base da cana-de-açúcar, em termos de oscilação do segmento e da faca, e a força de reação do segmento com a superfície.

\section{MATERIAL E MÉTODOS}

O estudo foi desenvolvido no Laboratório de Protótipos da Faculdade de Engenharia Agrícola - FEAGRI/UNICAMP.

A elaboração dos desenhos, o projeto e determinação das propriedades de massa das peças, foram obtidos através do programa Mechanical Desktop R4.0®. A simulação e a geração dos gráficos, foram desenvolvidas no programa computacional Matlab R6.1®.
Para simulação do protótipo virtual, composto de três peças (Figura 1), utilizou-se a rotina de programação proposta por Oliveira (2003) solucionando, de forma numérica e simultânea, um sistema de dezoito equações algébricas (Eq. 1).

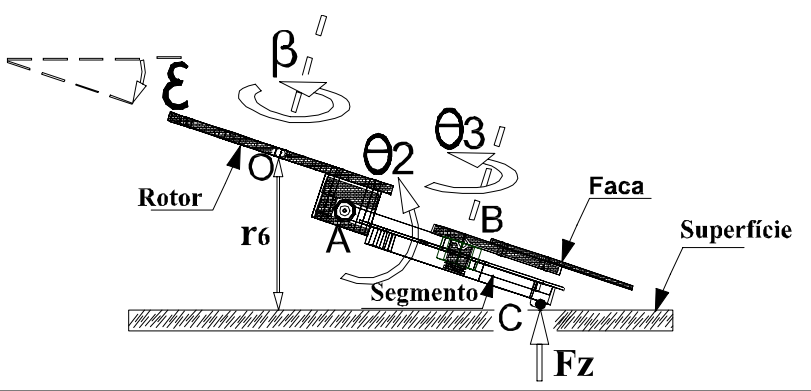

Figura 1. Esquema do protótipo bi-articulado de corte e varredura: $\varepsilon$ : inclinação do rotor; $\beta$ : giro do rotor; $\theta_{2}$ : oscilação do segmento no plano vertical; $\theta_{3}$ : oscilação da faca no plano horizontal; O: centro de massa do rotor; $\mathrm{r}_{6}$ : altura do rotor até a superfície; A: vínculo entre o segmento e o rotor; B: vínculo entre a faca e o segmento e, C: ponto onde surge a força reação Fz pelo contato entre o segmento e a superfície durante o giro $\beta$ do rotor.

As equações algébricas do sistema são as reações e os momentos nos vínculos entre as peças e, duas equações de movimento (mecanismo com 2 graus de liberdade). O primeiro movimento, denominado $\theta_{2}$, é a oscilação do segmento no plano vertical que, por sua vez é uma das variáveis que estão sendo analisadas neste trabalho e está relacionada à varredura de plantas acamadas sobre a superfície; o segundo movimento estudado, denominado $\theta_{3}$, é a oscilação da faca e está relacionado não autoproteção da faca, mediante a presença de obstáculos, como pedras e tocos, durante a operação do cortador. Por fim, simulou-se a força Fz de interação do segmento com a superfície, que se relaciona com a movimentação de terra e contaminação do material colhido.

As simulações foram efetuadas nas rotações do cortador de 400 e $600 \mathrm{rpm}$, inclinações do rotor de -10 e $-12^{\circ}$ (giro negativo no sentido horário) e nas alturas do rotor com a superfície de 120, 130 e $140 \mathrm{~mm}$. As condições para simulação foram realizadas de forma a se obter os máximos valores dos ângulos de varredura de plantas acamadas sobre a superfície, para o protótipo em questão (Oliveira et al., 2005) e por meio dos estudos com corte de base, realizados por Kroes \& Harris (1994).

Da concepção inicial do projeto do protótipo (Figura 1) até a construção da unidade experimental (Figura 2) para posterior validação do modelo, o programa de simulação sofreu aprimoramentos gradativos ao longo da pesquisa (Oliveira et al., 1999; Oliveira et al., 2001).

\section{RESULTADOS E DISCUSSÃO}

Os resultados da Tabela 1 apresentam o comportamento das variáveis amplitude de oscilação do segmento $\theta_{2}$ e a força de reação $\mathrm{Fz}$, atuante entre a superfície varrida e o segmento na direção perpendicular ao plano horizontal. Os valores da amplitude do ângulo $\theta_{2}$ diminuem com o aumento da velocidade de rotação de 400 para $600 \mathrm{rpm}$ e com o aumento 


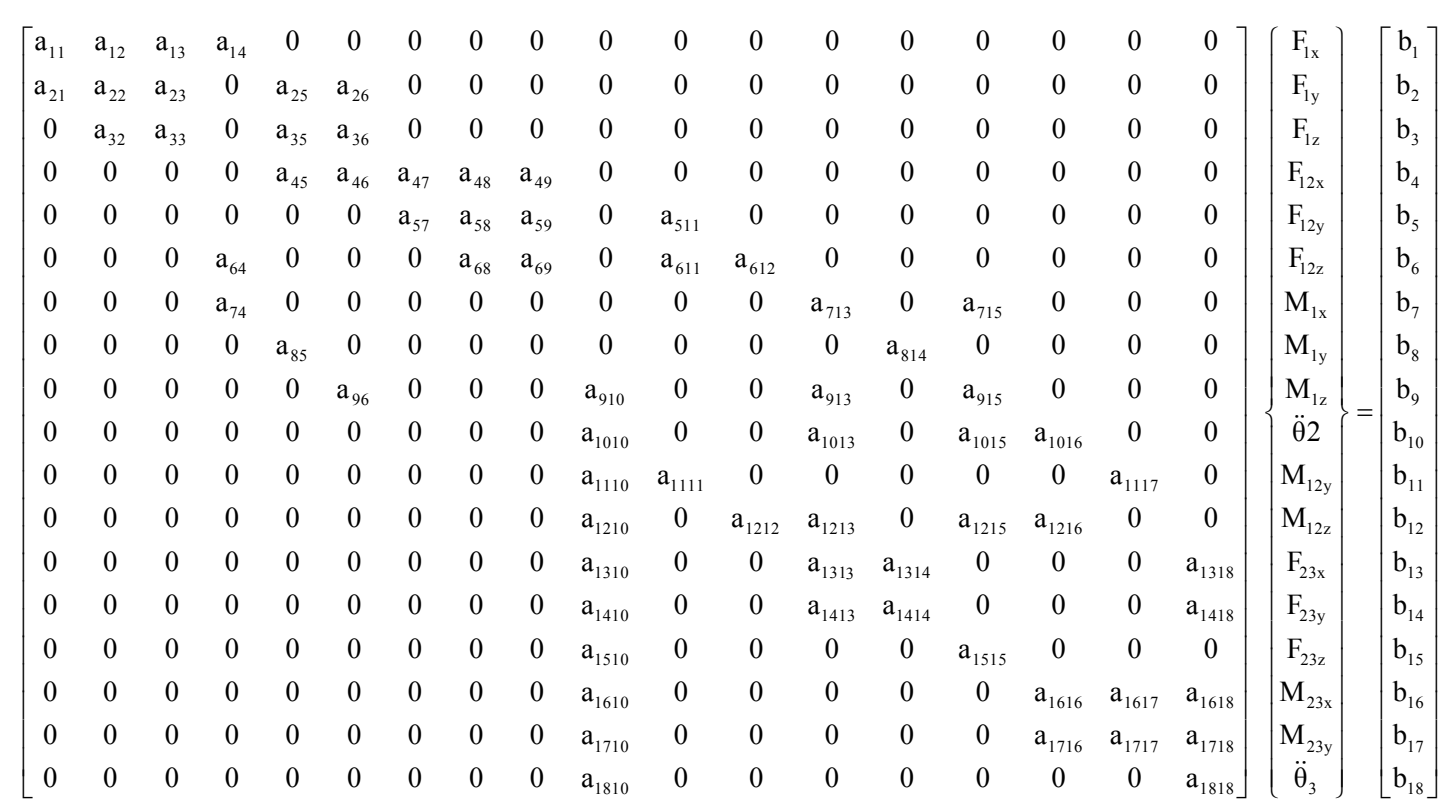

em que:

$\mathrm{a}_{1} \ldots \mathrm{a}_{1818}$ - coeficientes da matriz

$\mathrm{F}_{1 \mathrm{X}}, \mathrm{F}_{1 \mathrm{Y}}, \mathrm{F}_{1 \mathrm{Z}}$ - componentes do vetor $\overrightarrow{\mathrm{F}}_{1}$, que é a força de reação do rotor com a flange do motor hidráulico, $\mathrm{N}$

$\mathrm{F}_{12 \mathrm{X}}, \mathrm{F}_{12 \mathrm{Y}}, \mathrm{F}_{12 \mathrm{Z}}$ - componentes do vetor $\overrightarrow{\mathrm{F}}_{12}$, que é a força de reação no vínculo do segmento com o rotor, $\mathrm{N}$

$\mathrm{M}_{1 \mathrm{X},}, \mathrm{M}_{1 \mathrm{Y}}, \mathrm{M}_{1 \mathrm{Z}}$ - componentes do vetor $\mathrm{M}_{1}$, que é o momento de reação do rotor com a flange do motor hidráulico, $\mathrm{N} \mathrm{m}$ $\ddot{\theta}_{2}$ - aceleração (escalar) angular absoluta do segmento, $\operatorname{rad~s}^{2^{-1}}$

$\mathrm{M}_{12 \mathrm{Y}}, \mathrm{M}_{12 \mathrm{Z}}$ - componentes do vetor $\overrightarrow{\mathrm{M}}_{12}$, que é o momento de reação do segmento com o rotor, $\mathrm{N} \mathrm{m}$

$\mathrm{F}_{23 \mathrm{X}}, \mathrm{F}_{23 \mathrm{Y}}, \mathrm{F}_{23 \mathrm{Z}}$ - componentes do vetor $\overrightarrow{\mathrm{F}}_{23}$, que é a força de reação no vínculo da faca com o segmento, $\mathrm{N}$ $\ddot{\theta}_{3}$ - aceleração (escalar) angular absoluta da faca,

$\mathrm{M}_{23 \mathrm{X}}, \mathrm{M}_{23 \mathrm{Y}}$ - componentes do vetor, que é o momento de reação da faca sobre o segmento, $\mathrm{N} \mathrm{m}$ - termos independentes

do posicionamento em altura do rotor, de 120 para $140 \mathrm{~mm}$. A mudança no ângulo de inclinação do rotor de -10 para -12 graus, causou aumento da amplitude do ângulo $\theta_{2}$. Os valores máximos da força $\mathrm{Fz}$, resultantes do contato com a su-

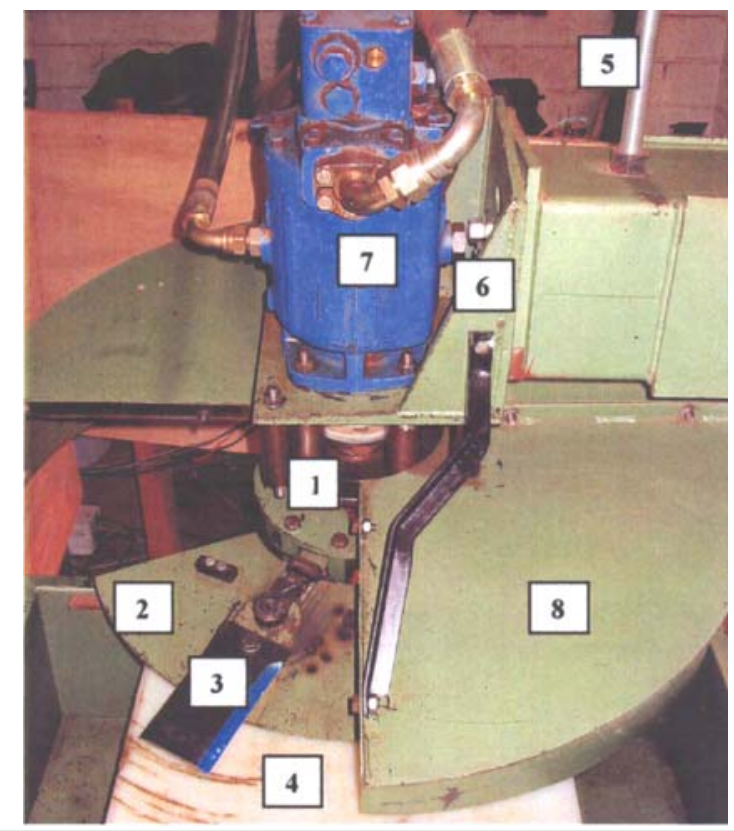

Figura 2. Unidade experimental: (1) rotor; (2) segmento; (3) faca; (4) superfície; (5) fuso para ajuste de altura $r_{6}$ do cortador; (6) dispositivo para ajuste angular $\varepsilon$ do rotor; (7) motor hidráulico e (8) cobertura de proteção
Tabela1. Comportamento de movimento do cortador nas condições ensaiadas*

\begin{tabular}{|c|c|c|c|c|}
\hline \multirow{2}{*}{ Variáveis } & \multirow{2}{*}{$\varepsilon$} & \multirow{2}{*}{$\mathbf{r}_{6}$} & \multicolumn{2}{|c|}{ Valores simulados } \\
\hline & & & $400 \mathrm{~min}^{-1}$ & $600 \mathrm{~min}^{-1}$ \\
\hline \multirow{6}{*}{ Amplitude $\theta_{2}$ (graus) } & \multirow{4}{*}{-10} & 120 & 7,4 & 5,7 \\
\hline & & 130 & 6,7 & 4,2 \\
\hline & & 140 & 3,2 & 2,1 \\
\hline & & 120 & 9,5 & 7,3 \\
\hline & \multirow{2}{*}{-12} & 130 & 7,6 & 5,4 \\
\hline & & 140 & 5,1 & 4,3 \\
\hline \multirow{6}{*}{ Força Fz (N) } & \multirow{4}{*}{-10} & 120 & 1777 & 2074 \\
\hline & & 130 & 1200 & 1697 \\
\hline & & 140 & 941 & 1075 \\
\hline & & 120 & 1916 & 2654 \\
\hline & \multirow[t]{2}{*}{-12} & 130 & 1601 & 2069 \\
\hline & & 140 & 1320 & 1610 \\
\hline
\end{tabular}

* Condições utilizadas na simulação: passo de integração de $0,0008 \mathrm{~s}$; massa do rotor $=4,2 \mathrm{~kg}$; massa do segmento $3,9 \mathrm{~kg}$, massa da faca $1,5 \mathrm{~kg}$ e constante elástica da superfície de $17^{*} 10^{4}$ $\mathrm{N} \mathrm{m}{ }^{-1}$

perfície, aumentam com o incremento da velocidade de rotação, de 400 para $600 \mathrm{rpm}$. Comportamentos similares aconteceram com a redução da altura de posicionamento do rotor de 140 para $120 \mathrm{~mm}$ e com o aumento da inclinação do disco, de -10 para $-12^{\circ}$.

Desta forma, um aumento de $200 \mathrm{~min}^{-1}$ na velocidade ou redução de altura de $10 \mathrm{~mm}$ ou, ainda, um aumento na inclinação do rotor de $2^{\circ}$, apresentaram efeitos da mesma ordem 
de grandeza sobre a força $\mathrm{Fz}$, de aproximadamente $410 \mathrm{~N}$. Nota-se que a magnitude de $\theta_{2}$, variável relacionada à varredura, foi elevada, variando de 2,1 a $9,5^{\circ}$, porém essas amplitudes acontecem na posição angular ocasião em que o segmento e a faca estão inoperantes, ou seja, opostos à região frontal do cortador, não causando efeito algum, em termos de corte e varredura.

Em análise das variáveis velocidade de rotação, inclinação e altura do rotor, Oliveira (2003) verificou que a varredura do protótipo aumenta com a velocidade de rotação e com a incli-
A.

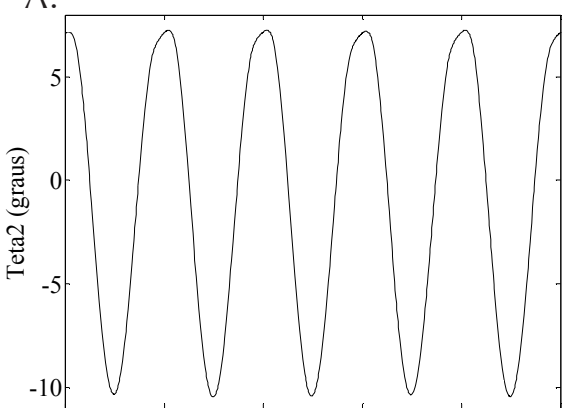

E.

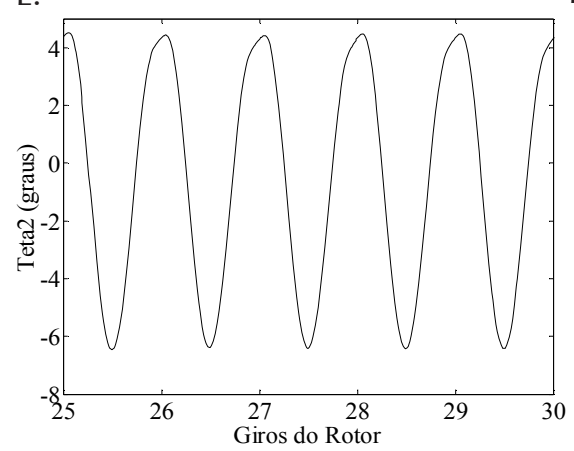

B.

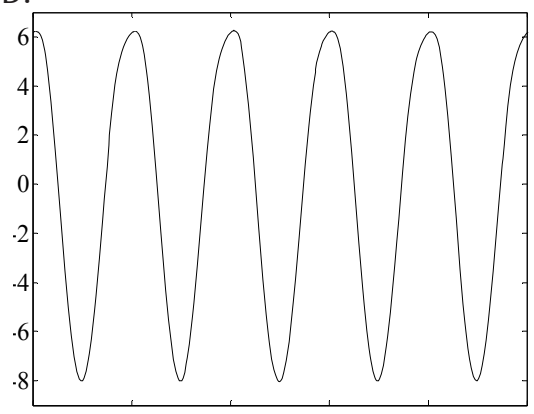

F.

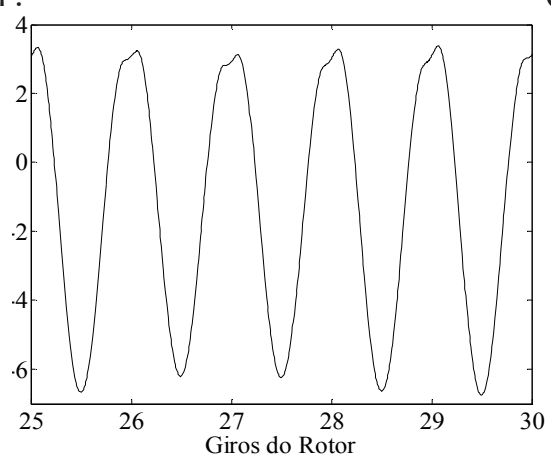

D.

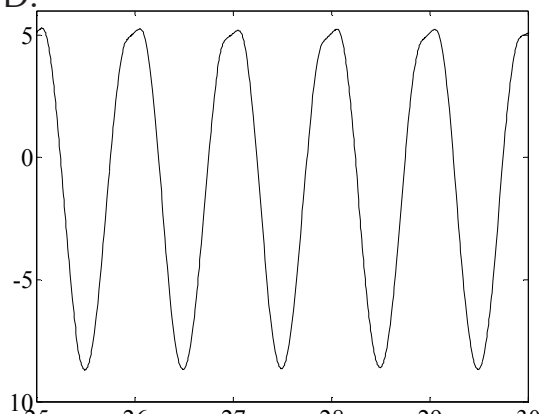

G.

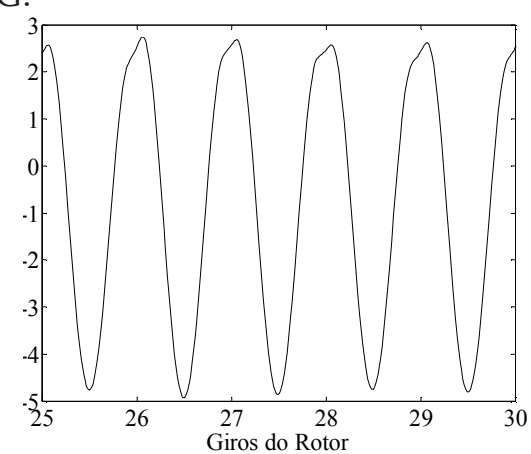

Figura 3. Oscilação vertical do segmento com inclinação do rotor de $-12^{\circ}$ : (A) velocidade do rotor de 400 min $^{-1}$ e altura $\mathrm{r}_{6}$ de $120 \mathrm{~mm}$; (B) velocidade de 600 min${ }_{1}^{1}$ e altura $\mathrm{r}_{6}$ de $120 \mathrm{~mm}$; (C) velocidade de $400 \mathrm{~min}^{-1}$ e altura $\mathrm{r}_{6}$ de $130 \mathrm{~mm}$; (D) velocidade de $600 \mathrm{~min}^{-1} \mathrm{e} \mathrm{r}_{6}$ de $130 \mathrm{~mm}$; (E) velocidade do rotor de $400 \mathrm{~min}^{-1} \mathrm{e} \mathrm{r}_{6}$ de $140 \mathrm{~mm}$ e (F) velocidade de $600 \mathrm{~min}^{-1} \mathrm{er}_{6}$ de $140 \mathrm{~mm}$

A.

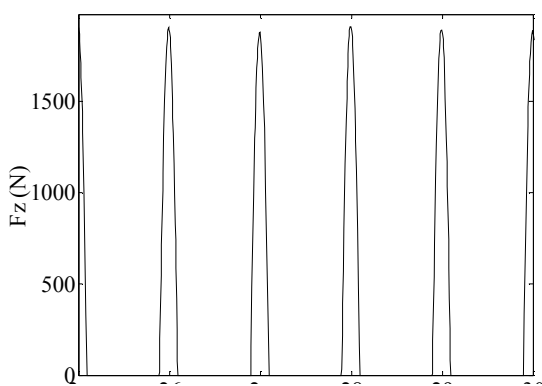

E.

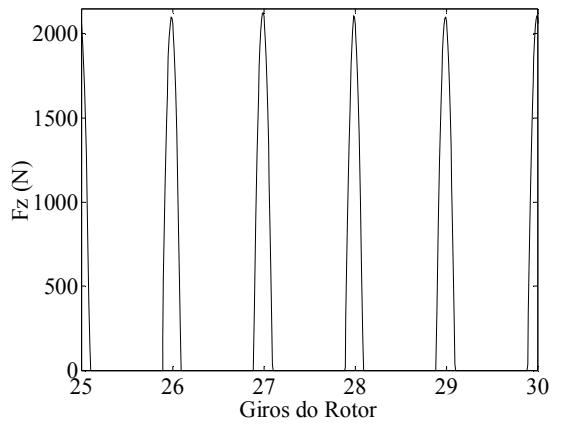

B.

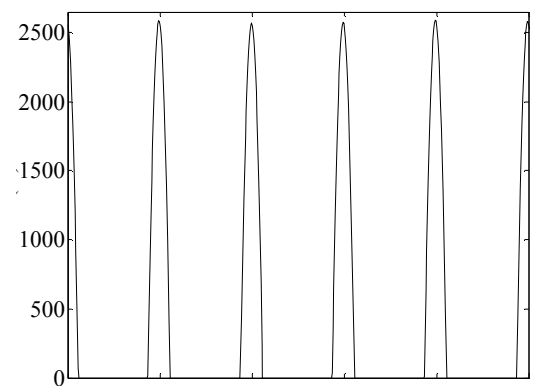

F.

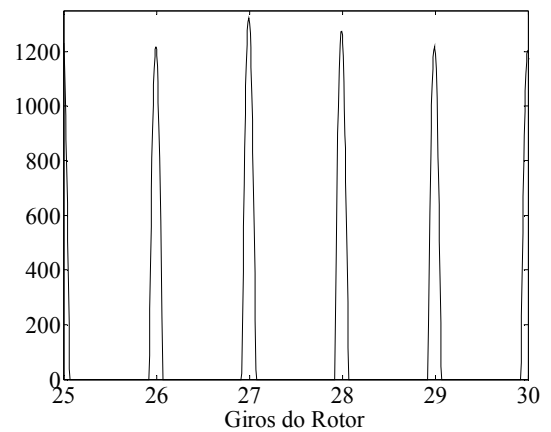

D.

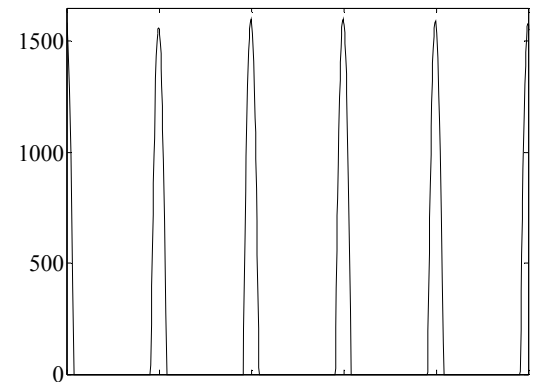

G.

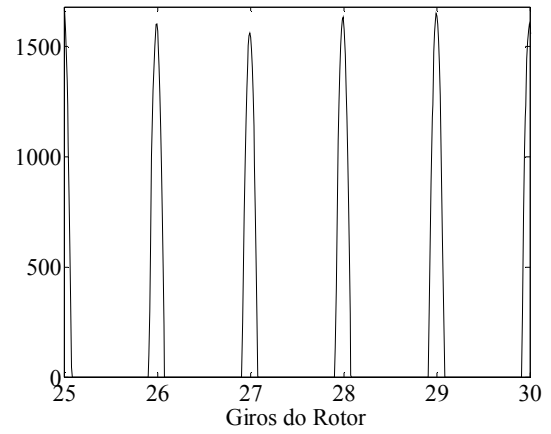

Figura 4. Reação vertical da superfície de varredura na inclinação do rotor de -12 graus: (A) velocidade do rotor de 400 min ${ }^{-1}$ e altura $\mathrm{r}_{6}$ de 120 mm; (B) velocidade do rotor de $600 \mathrm{~min}^{-1}$ e altura $\mathrm{r}_{6}$ de $120 \mathrm{~mm}$; (C) velocidade do rotor de $400 \mathrm{~min}^{-1}$ e altura $\mathrm{r}_{6}$ de $130 \mathrm{~mm}$; (D) velocidade de $600 \mathrm{~min}^{-1}$ e $\mathrm{r}_{6}$ de $130 \mathrm{~mm}$; (E) velocidade de $400 \mathrm{~min}^{-1}$ e $_{6}$ de $140 \mathrm{~mm}$ e (F) velocidade de $600 \mathrm{~min}^{-1}$ e $_{6}$ de $140 \mathrm{~mm}$ 
nação do rotor mas diminui com o incremento da altura do rotor.Uma diminuição da força de reação Fz, variável associada à movimentação e incorporação de terra ao material colhido, é conseguida com a diminuição da inclinação do rotor e com o aumento da altura com a superfície; entretanto, este fato acarreta diminuição dos índices de varredura (Oliveira et al, 2005).

Uma diminuição da amplitude de oscilação do ângulo $\mathrm{q}_{2}$ poderia ser conseguida com o aumento da relação entre a freqüência de excitação (rotação do mecanismo) e a freqüência natural do segmento. Esta alteração demandaria uma redução da freqüência natural, que poderia ser conseguida com o aumento da massa do segmento, porém esta alteração deve resultar em aumento da força de reação Fz com a superfície, cuja magnitude deve ser quantificada para verificar se é aceitável, em termos da compactação do solo. Esta análise conjunta das amplitudes de $\mathrm{q}_{2}$ e Fz só pode ser feita com dados específicos do comportamento mecânico de uma superfície de solo (Braunbeck, 2003). A magnitude média da força Fz foi de $1661 \mathrm{~N}$ que, apesar de elevada, deve apresentar redução drástica com o segmento, interagindo com a superfície do solo, devido à rigidez menor quando comparado com a superfície aqui simulada (Oliveira, 2003).

A oscilação $\theta_{3}$ da faca nas rotações de 400 e 600 rpm apresenou valores de amplitudes desprezíveis durante a simulação; isto é, comportamento consistente com a atual condição de avaliação do protótipo que desconsidera o corte da canade-açúcar.

A oscilação do segmento e a força de reação na inclinação de $12^{\circ}$, rotações de 400 e $600 \mathrm{rpm}$ e nas alturas de 120 , 130 e 140 mm, são representadas nas Figuras 3 e 4 . A simulação do protótipo é representada entre as 25 e $30^{\mathrm{a}}$ voltas do rotor, situação na qual já se tem estabilidade do movimento do mecanismo.

\section{CONCLUSÕES}

1. O modelo de simulação atendeu satisfatoriamente o dimensionamento da unidade experimental de corte e varredura.

2. As elevadas amplitudes de oscilação do segmento acontecem em uma região em que não ocorrem corte nem varredura dos colmos acamados.

3. Mudanças nas variáveis de projeto estudadas com o intuito de redução da força de reação com a superfície, causariam diminuição no índice de varredura, além de demandar um esforço não justificado na atual fase de pesquisa; considerando-se que o objetivo final do mecanismo será a varredura de superfícies de solos agrícolas.

\section{AGRADECIMENTOS}

Ao Conselho Nacional de Desenvolvimento Científico e Tecnológico (CNPq) pela concessão da Bolsa de Estudos, e à Fundação de Amparo à Pesquisa do Estado de São Paulo (FAPESP) pelo apoio financeiro, com vistas à construção da unidade experimental.

\section{LITERATURA CITADA}

Braunbeck, O.A. Proposta brasileira de colheita mecanizada. In: Seminário soluções e novidades na mecanização da canade-açúcar, 1, 1999, Ribeirão Preto. Anais... 1999 Ribeirão Preto: IDEA, 1999. p.56-60.

Braunbeck, O.A. Varredura do solo na colheita de cana-deaçúcar utilizando disco com segmentos articulados: modelagem e validação experimental. Campinas: UNICAMP. 2003, p.21. Relatório de Pesquisa FAPESP: Processo 2001/05910-8

Braunbeck, O.A.; Bauen, A.; Rosillo-Calle, F.; Cortez, L.A. Prospects for green cane harvesting and cane residue use in Brazil. Biomass and Bioenergy. England, v.17, n.1, p.495506, 1999.

Braunbeck, O.A.; Magalhães, P.S.G. Seguimento do perfil do solo no corte e/ou levantamento de produtos agrícolas rasteiros. Revista Brasileira de Engenharia Agrícola e Ambiental, Campina Grande, v.6, n.1, p.151-158, 2002.

Kroes, S.; Harris, H.D. Effects of cane harvester base cutter parameters on the quality of cut. Proceedings of Australian Society of Sugar Cane Technologists, Mackay, p.169177, 1994.

Magalhães, P.S.G.; Braunbeck O.A. Colheita de cana-de-açúcar: atualidade e perspectiva. In: Congresso de Ingeniería Rural y Mecanización Agraria en el Ambito Latinoamericano, 3, 1998, La Plata. Anais...La Plata: ALIA, 1998. p.262-271.

Oliveira, C.A.A. Modelagem e validação experimental de um cortador basal com discos segmentados. Campinas: UNICAMP, 2003. 110p. Tese Doutorado

Oliveira, C.A.A.; Braunbeck, O.A., Volpato, C.E.S. Simulação dinâmica de um mecanismo de disco segmentado para o corte e alimentação de gramíneas em operações de colheita. In: Congresso Brasileiro de Engenharia Agrícola, 28, 1999, Pelotas. Anais... Pelotas: SBEA, 1999. CD Rom

Oliveira, C.A.A.; Braunbeck, O.A., Volpato, C.E.S. Uso de modelagem e otimização no aprimoramento da varredura de um rotor de corte basal com movimento vertical das facas. In: Congresso Brasileiro de Engenharia Agrícola, 30, 2001, Foz do Iguaçu. Anais... Foz do Iguaçu: SBEA, 2001. CD Rom

Oliveira, C.A.A.; Braunbeck, O.A.; Volpato, C.E.S. Simulação da varredura da superfície por um cortador de base segmentado. Engenharia Agrícola. Jaboticabal, v.25, n.3, p.774-782, 2005.

Ridge, D.R.; Dick, R.G. Soil intake with cane during chopper harvesting: minimizing the problem. Proceedings of Australian Society of Sugar Cane Technologists, Mackay, v.14, p.25-30, 1992.

Volpato, C.E.S.; Braunbeck, O.A.; Oliveira, C.A.A. Desenvolvimento e avaliação de um protótipo de cortador base para colhedoras de cana-de-açúcar. Revista Brasileira de Engenharia Agrícola e Ambiental, Campina Grande, v.6, n.2, p.345-348, 2002. 